\title{
Lab@Home: Individualisierte Computerpraktika
}

\author{
F. M. Arnold, J.-O. Joswig*
}

Theoretische Chemie, Fakultät für Chemie und Lebensmittelchemie, TU Dresden

\begin{abstract}
Die im Jahr 2020 aufgetretene Pandemie bedingte auch an den Universitäten einen Lockdown und die Verlagerung der Lehre in den digitalen Raum. Im Bereich der Studiengänge Chemie und Lebensmittelchemie ist dies nur teilweise möglich. Insbesondere die Laborpraktika vermitteln Kernkompetenzen, die nicht anders als in Präsenz erworben werden können. Computerpraktika hingegen können mit guter Konzeption an den heimischen Computer verlagert werden. Wir stellen hier unser Konzept vor, das es möglich gemacht hat, Computerversuche aus den Bereichen der Quantenchemie und Statistischen Thermodynamik als Lab@Home-Computerpraktikum durchzuführen. Individualisierte Aufgabenstellung, kontrollierte Vorproduktion der numerischen Ergebnisse, fortlaufende Kommunikation mit den Studierenden und umfangreiche Nutzung digitaler Lehrmethoden waren dabei die entscheidenden Grundlagen für die erfolgreiche Durchführung.
\end{abstract}

The pandemic that occurred in 2020 also caused a lockdown at universities and the relocation of teaching to the digital space. In the area of the Chemistry and Food Chemistry degree programmes, this is only partially possible. The laboratory courses in particular convey core skills that cannot be acquired in any other way than in presence. Computer-lab courses, on the other hand, can be relocated to the home computer with a good underlying concept. We present our concept here, which has made it possible to conduct computer experiments from the fields of quantum chemistry and statistical thermodynamics as a Lab@Home computer-lab courses. Individualised tasks, controlled pre-production of numerical results, continuous communication with the students and extensive use of digital teaching methods were the decisive foundations for successful implementation. 


\section{Einleitung}

Ein Teil der Ausbildung in den Studiengängen Chemie und Lebensmittelchemie beschäftigt sich mit dem Fachgebiet der Theoretischen Chemie. Dieser Teil vermittelt die Grundlagen der Quantenmechanik, verschiedene Modelle zur Berechnung von molekularen Eigenschaften sowie Methoden der Elektronenstrukturrechnung. Bedingt durch die Interdisziplinarität dieses Gebiets sind die Studierenden mit Problemstellungen aus Physik, Mathematik und Computeranwendung konfrontiert, die alle zur Bearbeitung chemischer Fragestellungen benötigt werden. Wir haben die Erfahrung gemacht, dass die Vermittlung dieser Zusammenhänge durch praktische Übungen (HandsOn-Kurse) gut funktioniert und schon früh entsprechende Computerexperimente entworfen und durchgeführt [1].

Normalerweise fand dieses Computerpraktikum unter Aufsicht zu festgelegten Zeiten in einem gut ausgestatteten Computer-Pool statt. Durch die im Jahr 2020 aufgetretene Pandemie und die Lockdowns waren allerdings Präsenzveranstaltungen gar nicht bzw. nur unter erschwerten Umständen durchführbar. Wir haben uns daher schon früh entschlossen, diesen Bereich unserer Lehrveranstaltungen nach Hause zu verlegen: als Lab@Home. Zugute kam uns dabei die Tatsache, dass heutzutage alle Studierenden mit Computerhardware ausgerüstet sind und wir keine praktischen Versuche im Labor durchführen müssen.

Wir stellen in diesem Artikel unser Konzept der Überführung dieser Computerversuche in individualisierte Versuche vor, die im Homeoffice durchgeführt werden konnten. Die Planung für das Sommersemester 2020 erfolgte innerhalb von 14 Tagen, nachdem die TU Dresden in den Notbetrieb gegangen war. Wir haben unser Konzept, das nun bereits im zweiten Jahr erfolgreich läuft, stetig weiterentwickelt und an individuelle Gegebenheiten verschiedener Lehrveranstaltungen angepasst.

\section{Computerpraktika}

Unser vorliegendes Konzept haben wir auf zwei Praktika der Studiengänge Bachelor Chemie und Lebensmittelchemie angewendet.
Beide Praktika sind in den Modul-Kanon der Physikalischen Chemie (PC) eingebunden und sollen hier kurz beschrieben werden: Das Modul PC2 ("Theorie der chemischen Bindung") findet als Pflichtmodul beider Studiengänge im dritten Semester statt und beschäftigt sich mit den Grundlagen der Quantenmechanik (Schrödinger-Gleichung, Teilchen im Kasten, harmonischer Oszillator, Wasserstoffatom, Molekülorbital-Theorie, Hückel-Theorie) sowie Grundlagen der Elektronenstrukturrechnungen, wie Hartree-Fock-Methode und Dichtefunktionaltheorie. Neben der Vorlesung und einer Seminarreihe wird der Inhalt vor allem im PC2-Computerpraktikum vermittelt, das fünf Computerversuche beinhaltet. Deren Themengebiete sind: (1) Atomorbitale, (2) Ionisierungspotential, (3) Molekülorbitaltheorie, (4) Hückel-Theorie und (5) Schwingungsspektren.

Im sechsten Semester nehmen die Studierenden des Bachelorstudiengangs Chemie außerdem am Pflichtmodul PC3 („Spezielle Physikalische Chemie") teil, das sich mit den Teilgebieten Photochemie, Elektrochemie, Theoretische Chemie und Statistische Thermodynamik beschäftigt. Auch hier gibt es neben den Vorlesungen und einer Seminarreihe ein Praktikum, das aus zwei gleich großen Teilen besteht: einem Laborpraktikum, das die Teilgebiete Photochemie und Elektrochemie abdeckt, und einem Teil mit Computerexperimenten, die sich mit den Teilgebieten Theoretische Chemie und Statistische Thermodynamik beschäftigen.

Beiden Computerpraktika gemeinsam sind deren Modalitäten: Jeder der fünf (PC2) bzw. drei Versuche (PC3) wird durch ein versuchsbezogenes Seminar vorab eingeleitet, in dem die wichtigsten Grundlagen wiederholt und Besonderheiten des Versuchs diskutiert werden. Anschließend gibt es für die Studierenden in einen Zeitraum von vier Tagen die Möglichkeit, einen elektronischen Eingangstest (Antestat) durchzuführen, um die Zulassung zur Durchführung zu erhalten. Die hierbei erreichte Note geht in die Endnote ein.

Für diesen Eingangstest gibt es innerhalb des vorgegebenen Zeitraums zwei Wiederholungsmöglichkeiten. Tabelle 1 fasst wichtige Kenngrößen beider Praktika zusammen. 
Tab. 1: Zusammenfassung wichtiger Merkmale der zwei Module, in denen Lab@Home-Computerpraktika durchgeführt wurden. Die Laufzeit bezieht sich auf die Semester unter Pandemie-Bedingungen.

\begin{tabular}{lll}
\hline & Modul PC2 & Modul PC3 \\
\hline Modultitel & Theorie der chemischen Bindung & Spezielle Physikalische Chemie \\
\hline Studiengänge & Chemie, Lebensmittelchemie & Chemie \\
Studierende (2020) & ca. 90 (ca.45 Zweiergruppen) & ca. 40 \\
Semester & 3. (Wintersemester) & 6. (Sommersemester) \\
Anzahl Versuche & 5 (+ Vorversuch) & 3 (+ Vorversuch) \\
Laufzeit (2020) & 13 Wochen & 8 Wochen \\
\hline
\end{tabular}

Der eigentliche Versuch findet zu einem festgelegten Termin in unserem Computerpraktikumsraum statt, in dem 20 Computerarbeitsplätze mit der notwendigen Software ausgestattet sind. Jeder Versuch wird in Zweiergruppen durchgeführt, die Ergebnisse werden sofort handschriftlich protokolliert, diskutiert und das Protokoll am Ende des Versuchstages abgegeben. Der zeitliche Aufwand für die Studierenden vor Ort beträgt bei guter Vorbereitung etwa vier Stunden pro Versuch.

\section{Herausforderungen}

Mit dem Beginn eines Lockdowns ungewisser Dauer im Frühjahr 2020 ging die Notwendigkeit einer Entscheidung einher, ob und wie das im Sommersemester angesetzte PC3-Computerpraktikum durchgeführt werden könnte. Insbesondere die Unsicherheit über die Dauer des Lockdowns ließ uns schnell zu der Entscheidung gelangen, das Computerpraktikum nach Hause zu verlegen. Damit konnte die Zeit des harten Lockdowns sinnvoll genutzt werden und es wurden die Zeitblöcke, die für dieses Praktikum vorgesehen waren, frei und konnten den Kollegen für die Durchführung der experimentellen Laborpraktika zur Verfügung gestellt werden. Diese Lösung fand in der Studierendenschaft und unter den Kollegen großen Anklang.

Da die Qualität der Ausbildung auch unter diesen außergewöhnlichen Bedingungen zu gewährleisten war, warf die Verlagerung des Computerpraktikums an den studentischen Rechner als virtuelles Lab@Home-Praktikum eine Reihe von Fragen auf, die im Vorfeld zu lösen waren:

- Ist die bisher verwendete ComputationalChemistry-Software geeignet, auch von un- erfahrenen Studierenden ohne direkte Betreuung sinnvoll angewendet zu werden?

- Wie ist die korrekte und schnelle Installation der Software auf unterschiedlicher Hardware mit verschiedenen Betriebssystemen zu gewährleisten?

- Wie kann eine (zeitliche) Benachteiligung einzelner Studierender durch leistungsschwache Hardware verhindert werden?

- Wie kann eine asynchrone, aber doch lückenlose Betreuung während der Durchführung der Computerversuche garantiert werden?

- Wie kann erreicht werden, dass sich alle Studierenden selbst mit dem Stoff beschäftigen und die notwendigen Arbeitsschritte eigenständig durchführen?

- Wie können im Homeoffice geschriebene Testate sinnvoll gestaltet werden?

\section{Lösungsansatz: Lab@Home}

Unser Lösungsansatz für diese Fragen war die Individualisierung des Lab@Home-Computerpraktikums. In diesem Abschnitt erläutern wir zunächst die Einzellösung zu den aufgeworfenen Fragestellungen des vorherigen $A b$ schnitts und geben im anschließenden Abschnitt einen Überblick über die Modalitäten des Lab@Home-Praktikums, die sich nach drei Semestern herauskristallisiert haben.

Die im Computerpraktikum verwendete Computational-Chemistry-Software war auch vor der Pandemie schon das Software-Paket ADF (Amsterdam Density Functional) [2,3]. Über Lehrlizenzen kann mit vielen ComputationalChemistry-Software-Herstellern verhandelt werden. Dieses Programm verfügt über eine 
grafische Benutzeroberfläche, auf der Moleküle einfach generiert und alle Parameter der durchzuführenden Berechnung in DropDown-Menüs eingestellt werden können (Abb. 1). Da diese Software auf den gängigen Betriebssystemen (MS Windows, Mac OS, Linux) läuft, war sie ideal für unseren Ansatz geeignet. Wir begleiten den Installationsprozess und die Bedienung durch einen MessengerDienst-Kanal, aber auch per E-Mail und gegebenenfalls durch Videokonferenzen. Im Allgemeinen treten hierbei keine größeren Probleme auf, da der Installationsprozess dieser kommerziell erhältlichen Software bereits für die drei gängigen Betriebssysteme optimiert ist.

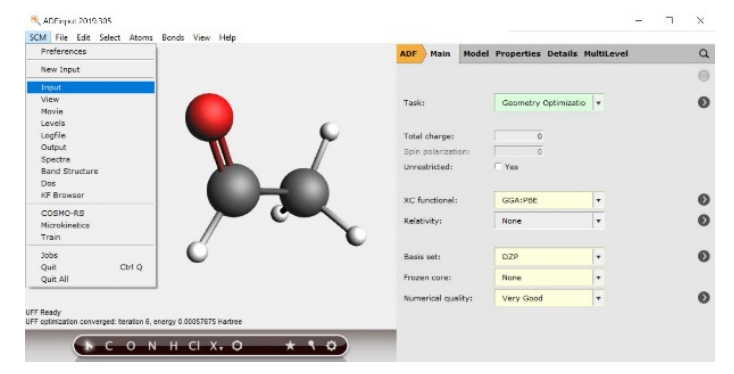

Abb. 1: Grafische Benutzeroberfläche des Programmpakets ADF [2,3]. Moleküle können intuitiv erstellt und Berechnungsparameter in verschiedenen Drop-Down-Menüs eingestellt werden.

Zusätzlich zur Möglichkeit des Lab@Home halten wir aber auch zwei vollständig eingerichtete Desktop-Workstations im Bereich des Campus in Einzelbüros bereit, so dass Studierende ohne die benötigten Hardware-Voraussetzungen die Versuche unter den gegeben Schutzmaßnahmen jederzeit durchführen könnten. Dadurch erreichen wir ein barrierefreies Computerpraktikum.

Die Installation der Software wird mittels eines Vorversuchs begleitet. In diesem Kurzversuch, zu dem kein Protokoll abgegeben werden muss, erhalten die Studierenden zusätzlich zur Installationsanleitung die numerischen Ergebnisse bestimmter Berechnungen, um diese zu reproduzieren und sich so mit der Software vertraut zu machen. Wir fragen bei diesem Versuch zusätzlich Informationen über die benötigten Rechenzeiten ab. Dadurch können wir die individuelle Hardware abschätzen und bei der Vergabe der Berechnungsparameter darauf achten, dass Studierende mit weniger leistungsstarker Hardware auch rechentechnisch weniger anspruchsvolle Datensätze zugeordnet bekommen.

Zur asynchronen, lückenlosen Betreuung während der Computerversuche verwenden wir den an der TU Dresden etablierten MessengerDienst [matrix] [4]. Der jeweilige PraktikumsChat-Kanal wird dabei ca. 12 Stunden pro Tag sowohl an Wochentagen als auch an Wochenenden betreut, so dass auf Studierendenseite im Falle von Fragen ein möglichst geringer Zeitverlust während der Bearbeitung der Versuche entsteht. Hierbei sind die Mitarbeiter der Professur für Theoretische Chemie involviert, die sich bei der Betreuung ablösen.

Die größte Herausforderung war die Qualitätssicherung: Während im Computerpool das Wechselspiel zwischen gewünschtem lehrreichem Austausch unter den Studierenden und unerwünschter Weitergabe von Lösungen relativ einfach überwacht werden kann, ist dies in einer Lab@Home-Situation nicht mehr möglich. Wir konnten dieses Problem umgehen, indem wir die Aufgabenstellungen individualisiert haben: Die Studierenden erhielten also individuelle Berechnungsparameter und zu untersuchende Moleküle zugeteilt. Mit diesen werden zwar qualitativ dieselben Ergebnisse erhalten, sie unterscheiden sich aber quantitativ (Abb. 2).

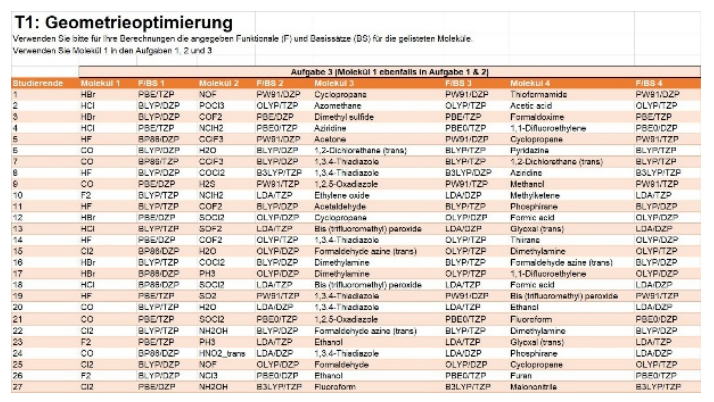

Abb. 2: Individuelle Zuteilung verschiedener Moleküle und Berechnungsparameter (Funktionale, Basissätze) an die Studierenden.

So konnten die Grundlagen für die didaktisch gewünschten Schlussfolgerungen gelegt werden, es mussten aber von allen Teilnehmern die entsprechenden Rechnungen selbständig durchgeführt, also individuelle Ergebnisse produziert werden. Auf diese Weise wurde zur eigenständigen Lösung der Aufgaben animiert 
und das Kopieren von Ergebnissen erschwert. Dieser Ansatz hat sich als sehr erfolgreich erwiesen. Zusätzlich wurden alle durch die Studierenden produzierten Datensätze vorab kontrolliert, so dass die Diskussion im Protokoll nicht mit inhaltlich falschen Ergebnissen vorgenommen wurde.

Natürlich hat die Individualisierung einen erheblichen Arbeitsaufwand vorab zur Folge, denn es mussten alle individuellen Datensätze zunächst vorproduziert werden. Dies war nicht nur als Grundlage für die Korrektur nötig, sondern diente auch dazu, bereits im Vorfeld didaktisch ungünstige Kombinationen von Molekülen und/oder Berechnungsparametern herauszufiltern. Praktisch umgesetzt wurde diese Vorproduktion der Ergebnisse durch die Mitarbeiter der Arbeitsgruppe Theoretische Chemie, die die Ergebnisse in geteilten Spreadsheets (Abb. 3) aus dem Homeoffice zusammentrugen und überprüften. Insgesamt wurden auf diese Weise mehrere tausend Datensätze produziert.

Während die kontrollierte Individualisierung des Computerpraktikums zur eigenständigen Beschäftigung mit dem Stoff führte, haben auf der anderen Seite Gemeinschaftsaufgaben eine konstruktive Zusammenarbeit zwischen den Studierenden initiiert.

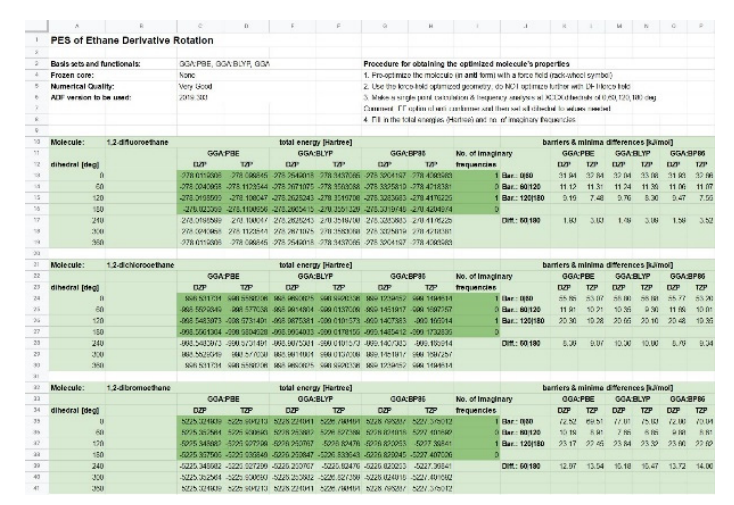

Abb. 3: Beispiel für ein geteiltes Spreadsheet, das von den Mitarbeitern der Arbeitsgruppe Theoretische Chemie befüllt wurde. Jede Rechnung wurde unabhängig voneinander von zwei Personen durchgeführt, um Fehler auszuschließen.

Zum Beispiel wurde von den Studierenden eine temperaturabhängige Darstellung einer Isomerenverteilung in ein geteiltes Spreadsheet eintragen, wobei jeder Teilnehmer ein Wertepaar beisteuerte (Abb. 4). Die entstehende Grafik wurde dann in den Protokollen diskutiert. Aus der sich entwickelnden Lösung konnten die Studierenden auch abschätzen, ob sie mit ihrem Ergebnis richtiglagen.

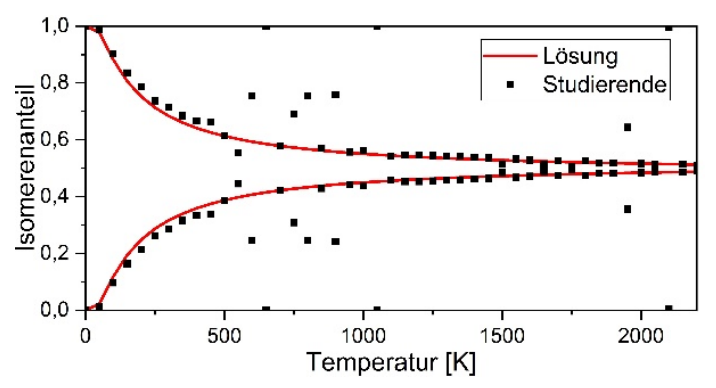

Abb. 4: Aus dem geteilten Spreadsheet erstelltes Diagramm, in das die Studierenden ihre Ergebnisse eintrugen. Die zwei roten Kurven zeigen das korrekte Ergebnis, die schwarzen Punkte die Ergebnisse der Studierenden mit einigen Abweichungen.

Auch dienten neu entwickelte Aufgaben mit aktuellem Themenbezug dazu, das Interesse der Studierenden über die normale Beschäftigung mit dem Stoff hinaus zu wecken. Dies schien gerade in einer Homeoffice-Situation wichtig, in der auch der direkte Austausch unter den Studierenden reduziert ist. Im dritten Semester (Modul PC2) wurden daher die molekularen Grundlagen des Treibhauseffektes, und damit ein aktuell diskutiertes Thema, in einen Versuch miteinbezogen. Hierbei ging es allein um die Vermittlung der physikalisch-chemischen Grundlagen, also naturwissenschaftlich verstandener Prozesse. Deren Verständnis lässt allen Studierenden die Möglichkeit, sich selbst in der politischen Debatte eine Meinung aufgrund naturwissenschaftlicher GesetzmäBigkeiten zu bilden.

Die gesamte Kommunikation verlief elektronisch. Das bedeutete, dass auch die Protokolle in elektronischer Form in Zweiergruppen (Modul PC2) bzw. allein (Modul PC3) eingereicht wurden.

Schließlich musste auch eine Lösung für die ursprünglich durchgeführten Eingangstests gefunden werden. Eingangstests dienen dazu, den Lehraufwand vor Ort zu minimieren, denn nur gut vorbereitete Studierende können aus den Computerversuchen lernen und sie in der gegebenen Zeit durchführen. Durch das vollständige Verlagern ins Homeoffice war dies nicht mehr notwendig. Der Lerneffekt setzte spätestens während der Durchführung des Versuchs ein, und Studierende mit gutem Vor- 
wissen konnten die Aufgaben schneller bearbeiten als unvorbereitete Teilnehmer. Wir haben die Eingangstests daher in Ausgangstests umgewandelt, um den Lernerfolg zu überprüfen und zu belohnen.

Den Herausforderungen, die die Verlegung der Computerpraktika nach Hause mit sich bringt, kann also wie folgt begegnet werden:

- Eignung der Software: Das von uns verwendete Programmpaket ADF ist optimal für die Ansprüche des Lab@Home geeignet. Andere Software kann dies auch sein, wurde von uns jedoch nicht getestet. Es empfiehlt sich, auf einfache Installation und intuitive Bedienbarkeit zu achten. Die Möglichkeit des Erwerbs von Lehrlizenzen oder eine freie Lizensierung ist dabei von Vorteil.

- Korrekte Installation: Auch wenn kommerzielle Programme üblicherweise auf eine möglichst zuverlässige Installation ausgelegt sind, haben wir diese mit einem Vorversuch begleitet.

- Technische Gleichberechtigung: Abgefragte Rechenzeiten gaben einen Anhaltspunkt für die Leistungsfähigkeit der verwendeten Hardware. Diese konnte so in der Zuordnung der Datensätze berücksichtigt werden.

- Asynchrone, lückenlose Betreuung: Ein Messenger-Dienst bietet die optimale Möglichkeit, schnell auf Fragen der Studierenden zu antworten.

- Eigenständigkeit: Die Individualisierung der Aufgabenstellungen führt zur eigenständigen Beschäftigung mit dem Stoff. Gemeinschaftsaufgaben motivieren zusätzlich.

- Ausgangstests statt Eingangstests: Zur Überprüfung und Belohnung des Lernerfolgs wurden elektronische Abtestate durchgeführt, die in die Versuchs- bzw. Modulnote eingingen.

\section{Digitale Betreuung}

Nutzung einer Lernplattform: Spätestens mit dem Beginn der Pandemie wurden die Vorteile der Nutzung einer Lernplattform offenbar. Wir haben die sächsische Lernplattform OPAL bereits zuvor intensiv für unsere Lehre genutzt. Die folgenden Funktionen waren dabei besonders hilfreich:

- Studentische Registrierung: E-Mail-Kontakte mit den Studierenden waren so jederzeit möglich.

- Upload/Download-Ordner: Die Protokolle wurden per Upload durch die Studierenden abgegeben. Die bewerteten Protokolle konnten zum Download bereitgestellt werden.

- Elektronische Tests: Die Ausgangstests wurden mit Hilfe des Testwerkzeugs ONYX durchgeführt. Für Ausgangstests wurde keine Wiederholungsmöglichkeit gegeben. In wenigen Fällen wurde der Versuch mit einem mündlichen Kolloquium abgeschlossen.

- Forum: Zunächst wurde das in die Lernplattform integrierte Forum zur Beantwortung von Fragen genutzt. Der MessengerDienst hat sich aber als für diesen Zweck geeigneter erwiesen.

- Klausur: Abschlussklausuren wurden während der Pandemie als online-Klausuren mit individualisierten Aufgabenstellungen durchgeführt.

Synchrone/asynchrone digitale Vorlesungen: Alle Vorlesungen der Module wurden aufgezeichnet und waren während des gesamten Semesters über eine Videoplattform (Videocampus Sachsen [5]) des Bildungsportals Sachsen [6] online abrufbar. Dadurch konnte das klassische Vorlesungsformat mit 90 Minuten Frontalunterricht beibehalten werden, denn bei Bedarf konnten die Vorlesungen ganz oder teilweise nachträglich angesehen werden. Die Aufzeichnung erfolgte zum Teil aus dem Hörsaal mit minimaler Präsenz oder vom Laptop über einen Videostreaming-Dienst.

Online-Seminare mit Breakout-Räumen: Eine der größten Herausforderungen war es, die Aufmerksamkeit in Seminaren zu halten, die aus verschiedenen Gründen nicht aufgezeichnet wurden. Dies geschah hauptsächlich durch Unterbrechungen des Vortrags durch Break- 
out-Sessions, in denen die Studierenden in zufällig generierten Kleingruppen Aufgaben bearbeiten konnten. Zum Teil betraf dies schon Lösungsansätze der Computerversuche. Dadurch wurde Abwechslung erzeugt und zur eigenständigen Beschäftigung mit dem Stoff angeregt. Die Schwelle, Fragen zu stellen, war in Kleingruppen deutlich reduziert.

Lernvideos: Neben den Online-Seminaren wurden die wesentlichen Inhalte der Versuche in kurzen Videos zusammengefasst, um den Studierenden zu ermöglichen, sich auch nach der jeweiligen Seminarveranstaltung mit den Inhalten zu beschäftigen. Pro Versuch wurden zwei bis drei Videos von je 10 bis 20 Minuten Länge produziert und über die Videoplattform Videocampus Sachsen veröffentlicht.

Digitale Klausur: Eine der größten Herausforderungen war das Stellen einer digitalen Klausur. Während die technischen Voraussetzungen durch die Lernplattform OPAL und das Testwerkzeug ONYX gegeben waren, kam es hier auf eine gute Konzeption der Klausuraufgaben an. Wir haben uns für eine Open-BookKlausur entschieden (inkl. Internetnutzung), da die Verwendung unerlaubter Hilfsmittel unter den gegebenen Umständen nicht überprüfbar war. Die Fragestellungen wurden entsprechend angepasst, so dass eine Online-Suche nach Lösungen keine Ergebnisse ergab oder zu lange dauerte. Zusätzlich wurden auch hier die Aufgabenstellungen individualisiert und zufällig verteilt und sortiert, so dass auch eine nicht auszuschließende Kommunikation unter den Studierenden zu viel Zeit in Anspruch nehmen würde. Die Notenverteilung hat sich nicht von denen der Vorjahre unterschieden, die Quote an nichtbestandenen Prüfungen war jedoch leicht erhöht.

Kommunikation: Als entscheidender Punkt für den Erfolg des Praktikums hat sich die Kommunikation mit den Studierenden erwiesen. Hierbei schien der wichtigste Punkt eine schnelle Beantwortung von Fragen zu sein. Dies war auch der Grund, weswegen wir nach einem Semesterdurchlauf von der Benutzung eines Forums zur Benutzung eines MessengerDienstes übergegangen sind, da hier die Benachrichtungsfunktion bei neuen Inhalten technisch besser umgesetzt ist.
Zur Kommunikation gehörte auch, den Studierenden das Gefühl des Betreut-Seins zu geben: Wir haben daher nicht nur alle Termine, Modalitäten und Praktikumsmaterialien frühzeitig veröffentlicht, sondern auch freitags eine wöchentliche E-Mail mit den Terminen der kommenden Woche und weiteren kurzen Informationen versendet.

\section{Lab@Home-Schlüsselelemente}

Für die erfolgreiche Durchführung eines Lab@Home-Computerpraktikums haben sich für uns folgende Punkte als entscheidend herauskristallisiert:

- Individualisierbare Aufgabenstellungen (quantitativ verschieden, qualitativ analog).

- Gute und permanente elektronische Kommunikation mit den Studierenden.

- Unterstützung der Asynchronizität durch bereitgestellte Lernvideos und Vorlesungsmitschnitte.

- Durchführung elektronischer Tests auch als Vorbereitung auf digitale Klausuren.

- Bereitschaft der Verantwortlichen zu neuen und ungewohnten Lehr- und Bewertungsformaten.

Diese Punkte bedingen einen erhöhten Personal- und Zeitaufwand sowohl bei der Vorbereitung als auch bei der Durchführung. Wir haben abgeschätzt, dass der Aufwand pro Semester bei etwa 1.000 Mitarbeiterstunden, wahrscheinlich aber sogar höher liegt. Hierin ist die benötigte Zeit für gedankliche Vorbereitung und Konzeption nicht einbezogen. Ein gut eingespieltes, nicht zu kleines Team ist daher unbedingt von Nöten.

In kommenden Semestern wird der Aufwand sicherlich sinken, allerdings müssen zum Beispiel die numerischen Ergebnisse bei Software-Updates regelmäßig überprüft werden.

\section{Nachhaltigkeit und Diversität}

Computerpraktika können generell als nachhaltige Lehrform angesehen werden. Die eigenständige Beschäftigung mit dem Stoff fin- 
det ohne Chemikalienverbrauch und sogar unabhängig von vor Ort zur Verfügung stehenden Räumlichkeiten statt. Die Individualisierung brachte einen erhöhten Vorbereitungsund Betreuungsaufwand mit sich, da mehrere tausend Datensätze produziert und getestet werden mussten. Dieser Prozess kann durch erfahrene Helfer unter Verwendung von scripting zumindest teilweise automatisiert werden. Die Datensätze müssen zwar überprüft werden, sobald ein Software-Update der verwendeten Programme durchgeführt wurde. Im Prinzip stehen sie aber für weitere Jahrgänge zur Verfügung. Auch für zukünftige Präsenzveranstaltungen können sie genutzt werden, da dann zwar eine synchrone Lehrveranstaltung durchgeführt wird, jede teilnehmende Gruppe aber individuelle Aufgabenstellungen erhalten kann.
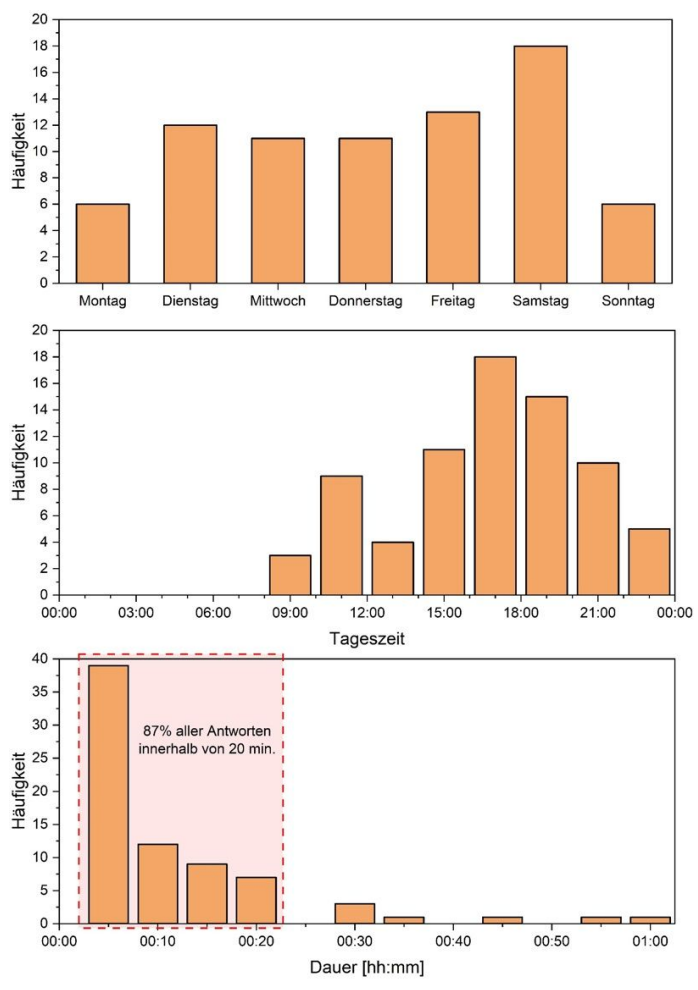

Abb. 5: Statistische Auswertung der Aktivitäten des Messenger-Dienst-Kanals für das Wintersemester 2020/21: Fragenhäufigkeit nach Wochentag (oben) und Tageszeit (Mitte) sowie Antwortzeit durch unsere Mitarbeiter (unten).

Insbesondere die statistische Auswertung des Praktikums-Kanals im Messenger-Dienst [matrix] zeigte, dass das Bearbeitungsverhalten der Studierenden stark vom typischen Tagesablauf mit Präsenzveranstaltungen abwich
(Abb.5). Die Lab@Home-Aufgaben wurden vermehrt am Wochenende und am Abend durchgeführt. Die Statistik zeigt auch, dass unsere Mitarbeiter es geschafft haben, ca. 90\% der gestellten Fragen unabhängig von Wochentag und Uhrzeit innerhalb von 20 Minuten zu beantworten.

Für Studierende, die sich mit besonderen Umständen auseinanderzusetzen haben, z.B. bei Krankheit oder Abwesenheit, ist die Möglichkeit, ein asynchrones Computerpraktikum zu absolvieren, sicherlich attraktiv. Mit dem gleichzeitigen Angebot des Durchführens vor Ort (durch bereitgestellte, buchbare Hardware im Bereich des Campus) sind unsere Lab@Home-Computerpraktika barrierefrei.

\section{Zusammenfassung und Ausblick}

Bedingt durch die im Frühjahr 2020 aufgetretene Pandemie und die daraus resultierenden Lockdowns waren wir gezwungen, ein neues Konzept für alle von uns durchgeführten Computerpraktika zu entwickeln. Mittlerweile laufen Lab@Home-Praktika bereits im dritten Semester erfolgreich. Der wichtigste Punkt bei der Entwicklung war die Aufrechterhaltung der Lehrqualität. Wir haben dies erreicht, indem wir die Aufgabenstellungen individualisiert haben. Zusätzlich haben wir für eine gute und permanente Kommunikation mit den Studierenden gesorgt. Hier hat sich ein MessengerDienst als vorteilhaft erwiesen. So war es möglich, den sich allein mit dem Stoff beschäftigenden Studierenden ein Betreuungsgefühl zu geben. Positiv aufgenommen wurden vor allem die Asynchronizität und die Barrierefreiheit. Zusätzlich zu den zu vermittelnden Themengebieten waren die Studierenden aber auch gezwungen, sich mit computertechnischen Fragestellungen auseinanderzusetzen (Programminstallation, vertiefte Nutzung von Tabellenkalkulationsprogrammen etc.), die innen auch außerhalb der Theoretischen Chemie zugutekommen.

\section{Danksagung}

Wir danken allen Beteiligten dieses Projekts: Thomas Heine und Antje Völkel, den Systemadministratoren und Doktoranden der Ar- 
beitsgruppe Theoretische Chemie, dem Zentrum für interdisziplinäres Lernen und Lehren (ZILL) der TU Dresden, insbesondere dem eLearning-Support, sowie den Studienjahrgängen 2017/2018 des Studiengangs Chemie und 2019 der Studiengänge Chemie und Lebensmittelchemie. Wir danken außerdem der Firma SCM für die Unterstützung mit ADF-Lehrlizenzen.

\section{Literatur}

[1] Computational Chemistry Workbook, T. Heine, J.-O. Joswig, and A. Gelessus, Wiley-VCH (2009), ISBN 9783-527-32442-2

[2] G. te Velde, F. M. Bickelhaupt, E. J. Baerends, C. Fonseca Guerra, S. J. A. van Gisbergen, J. G. Snijders and T. Ziegler, Chemistry with ADF, J. Comput. Chem. 22 (2001), 931, DOI: $10.1002 / j c c .1056$

[3] ADF 2021.1, SCM, Theoretical Chemistry, Vrije Universiteit, Amsterdam, The Netherlands, http://www.scm.com

[4] [matrix], Open-Source-Messengerdienst: https://matrix.org

[5] Videocampus Sachsen (gemeinsame Videoplattform sächsischer Universitäten, Hochschulen und der Berufsakademie Sachsen), betrieben von der BPS Bildungsportal Sachsen $\mathrm{GmbH}$ : https://videocampus.sachsen.de

[6] Bildungsportal Sachsen, Die sächsische E-LearningLandesinitiative, https://bildungsportal.sachsen.de 